\title{
Exotic diseases of dogs and cats at risk of importation to Ireland
}

\author{
Mark Goodfellow and Susan Shaw \\ Department Clinical Veterinary Science, University of Bristol, Langford House, Langford, North Somerset \\ BS40 5DU, United Kingdom \\ Changes in legislation that facilitate movement of companion animals within the European Union \\ will expose those animals to microbial and parasitic organisms currently exotic to Ireland. This paper \\ reviewed information on the exotic diseases most likely to be introduced to Ireland by travelling \\ dogs and cats: rabies, leishmaniosis, babesiosis, ehrlichiosis, anaplasmosis and dirofilariosis.
}

Irish Veterinary Journal

$58: 27 \mid-277$

\section{Introduction}

As a result of changes to European Union (EU) legislation facilitating the non-commercial movement of pet animals within the EU, it is expected that there will be an increase in the number of pets travelling to and from accredited countries. Many of these animals will be exposed to, and infected with, microbial and parasitic organisms currently exotic to Ireland, and veterinary surgeons will be required to diagnose, treat and prevent the exotic diseases caused by those organisms. This paper aims to review information on the most relevant diseases that may be acquired by travelling pets.

\section{Rabies \\ Rabies is a viral disease affecting the central nervous system of mammals. Dogs, cats, and wildlife species such as foxes and bats are important hosts for the virus, with dogs constituting the greatest risk to humans. The rabies virus is an enveloped RNA Lyssavirus, with multiple genotypes. Contamination of a bite wound with infected saliva accounts for the majority of cases. The incubation period ranges from ten days to four months (three to eight weeks in the dog and four to six weeks in the cat) and varies with the anatomical location (particularly, proximity to the spinal cord), degree of innervation at the site of infection, and the dose of virus inoculated.}

Pathogenesis and clinical signs

The virus multiplies in muscle fibres and connective tissue at the site of infection and may remain in situ for days to months. It then enters adjacent nerves, spreads to the central nervous system (CNS) and then distally along peripheral nerves to other parts of the body

Corresponding author:

Susan Shaw,

Department Clinical Veterinary Science,

University of Bristol,

Langford House,

Langford, North Somerset BS40 5DU, United Kingdom.

E-mail susan.e.shaw@bristol.ac.uk including the salivary glands. Salivary shedding of virus may occur five to 13 days before clinical signs appear in dogs, and for one to two days before clinical signs in cats. Damage to motor neurones causes flaccid paralysis and direct damage to CNS is compounded by immune-mediated damage.

Clinical signs are variable but commence with a "prodromal" stage of two to three days duration marked by a sudden temperament change that may be missed by the owner. Normally friendly dogs may hide while shy dogs may show a sudden display of affection. Hyperaesthesia may be present at the site of the bite wound. The prodromal stage is followed by one of two syndromes, referred to as "dumb" or "furious" rabies. "Dumb" rabies is more common in rabid dogs but some may alternate between "dumb" and "furious" forms. In "dumb" rabies, the clinical course is often shorter but, in both forms, death occurs three to seven days after the end of the prodromal stage. Dogs with the "dumb" syndrome may have an apprehensive appearance, may snap at imaginary objects although they will rarely bite unless provoked. In the "furious" syndrome, dogs become restless, irritable, pace continuously and may run aimlessly for long distances if not restrained. If restrained, they may display inappropriate, uncontrolled signs of "rage", attacking cage bars or furniture. In both syndromes, progressive hind limb weakness with ascending paralysis develops in association with cranial nerve palsies. Clinical signs include pupillary dilation and loss of the corneal reflex, whilst mandibular and glossal paralysis is manifested by profuse salivation and dysphagia. Laryngeal paresis may result in hoarse barking (dysphonia).

The signs shown by cats resemble those in dogs but the furious form occurs in about $75 \%$ of cases. The prodromal stage rarely lasts for more than 24 hours and the furious stage lasts for one to four days. Rabid cats display hypersensitivity to sensory stimuli and often retreat into hiding from which they may attack humans and other animals. Pupillary dilation, protrusion of claws and continuous hoarse vocalisation develop. Hind limb ataxia is followed by ascending paralysis and death.

Diagnosis

Rabies is a notifiable disease and all dogs and cats with compatible 
clinical signs (particularly if they have travelled overseas) should be isolated, securely confined and the appropriate government authority informed. There is obvious danger in handling suspect dogs and cats and premortem tests are not considered appropriate for rabies diagnosis in UK and Ireland. Diagnosis is made by postmortem histological examination of the brain at specialised facilities. Histologically, most cases show a necrotising encephalitis, ganglioneuritis, mononuclear cell perivascular cuffing, intracytoplasmic inclusions (Negri bodies), and the diagnosis is confirmed using direct fluorescent antibody testing of tissue which is both rapid and sensitive. Specific polymerase chain reaction (PCR) techniques have been developed also.

\section{Treatment and prevention}

No attempt should be made to treat suspected cases. Currently (2005), only killed vaccines are licensed for dogs and cats in the UK and Ireland and they induce a high level of protection against the disease. Other vaccines may be licensed in the future. Vaccination can be given from four weeks of age, assuming the dam has not been vaccinated. Booster vaccination is given every one to two years depending on the species being vaccinated and the vaccine used.

Rabies virus is inactivated by UV light, heat, detergents and disinfectants. In potential human exposure, immediate treatment of bite wounds with detergents, soap or quaternary ammonium compounds ( $1 \%$ to $4 \%$ solution) significantly reduces the risk of infection.

\section{Leishmaniosis}

Leishmaniosis is an important disease of dogs that is endemic in the Mediterranean areas of Europe, the Middle East and many tropical and subtropical areas of the world. In Europe, the aetiological agent of canine leishmaniosis is an intracellular protozoan parasite, Leishmania infantum, which is transmitted by sandflies of the genus Phlebotomus. In non-endemic areas of northern Europe, the occurrence of canine leishmaniosis is increasing rapidly due to increased mobility of infected dogs. This is particularly marked in countries where quarantine regulations have been relaxed for movement of dogs from leishmaniaendemic European countries. In addition, there are reports that potentially competent sandflies are being identified north of the traditional endemic areas such as in southern Germany; should temperatures rise in southern Ireland and England, conditions may become appropriate for the establishment of vectors. In several European countries, including the UK, $L$. infantum infections are already being reported in dogs that have never travelled to endemic areas (autochthonous infection) and canine leishmaniosis has spread extensively throughout North America in foxhounds without confirmed involvement of a recognised sandfly vector. Non-vector transmission has been confirmed in dogs receiving infected blood transfusions and amongst human intravenous drug users who share infected needles. Vertical and direct transmission is also suspected in some cases.

\section{Pathogenesis and clinical signs}

In endemic areas dogs become exposed almost immediately. Some will develop protective immunity, some remain sub-clinically affected and may relapse later, and others develop the classic clinical syndrome at a young age. The disease has a chronic waxing and waning course and the incubation period may vary from several months to years. The parasite stimulates an overactive and ineffective B lymphocyte response at the same time as suppressing macrophage activation. This allows spread of the parasites in the face of massive production of immunoglobulins and immune complexes. Both leishmania-specific and non-specific antibodies, including autoantibodies, are produced.

Non-specific signs of disease include a history of lethargy, weight loss, polydipsia, diarrhoea, and partial anorexia. Generalised lymphadenopathy is common. Cutaneous signs are of major significance and include periocular alopecia, hyperkeratosis of the footpads, excessive growth of claws and exfoliative dermatitis producing a characteristic silvery scale prominent on the dorsum of the nose, periocular region and pinnae. Ulcerative and nodular dermatitis may occur secondary to vasculitis and granulomatous inflammation. Polydipsia and weight loss due to protein-losing nephropathy are common, as are ocular signs due to panophthalmitis. Shifting lameness and joint swelling due to immune-mediated polyarthritis and Coombs'-positive anaemia may be present. Clinicopathological findings include marked proteinuria, hyperglobulinaemia (sometimes with monoclonal or biclonal spikes) and occasional antinuclear antibody positivity. Concurrent ehrlichiosis is common in endemic areas and care should be taken to eliminate coinfection in dogs that have bleeding diatheses.

\section{Diagnosis}

Along with typical clinical signs and a history of exposure, definitive diagnosis of leishmaniosis is based on microscopic identification of the parasite in stained (Giemsa or Leishman) aspirates of lymph node, bone marrow or spleen, or in skin biopsies. Immunohistochemistry may increase the sensitivity of detection in tissue biopsies. Many serological techniques are available including immunofluorescent antibody test (IFAT), and enzyme-linked immunosorbent assays (ELISA) and high titres or rising titres are compatible with active infection rather than exposure. Polymerase chain reaction (PCR) testing on bone marrow samples is sensitive and specific for leishmaniosis but because of tissue localisation has only $60 \%$ to $70 \%$ sensitivity on peripheral blood samples. Real time PCR testing on peripheral blood samples has increased sensitivity and quantification allows monitoring of therapy.

\section{Treatment and control}

The zoonotic potential of this disease is considered low without the presence of competent vectors but implications should be discussed with owners, particularly if there is an immuno-compromised individual in the household. The documented transmission of infection between dogs in the absence of the sandfly vector has unknown zoonotic implications.

Prior to therapy, the extent of systemic disease should be fully evaluated and appropriate supportive treatment given. Although other drugs may be used in some countries, treatment most commonly recommended is a combination of allopurinol and a pentavalent antimonial drug such as meglumine antimonate (Table I). In nonendemic areas, allopurinol is used initially with the addition of an antimonial course if there is poor response. Allopurinol treatment is continued on a long-term basis to maintain remission. Treatment could 
be suspended if serology and PCR on bone marrow are negative. The drug is inexpensive and major side effects with long-term therapy have not been reported. Concurrent treatment for ehrlichiosis may be necessary. Prognosis is dependent on the severity of organ dysfunction, particularly of the kidneys, at the time of diagnosis. If the dog is a treatment candidate, the prognosis for parasitological cure is guarded although the prognosis for control of the clinical expression of disease is good.

Vaccination is not yet available for leishmaniosis. Sandfly biting is reduced by using synthetic pyrethroids (permethrin and deltamethrin), and animals should be housed during peak sandfly activity.

\section{Feline leishmaniosis}

Natural infection and clinical disease in domestic cats caused by Leishmania spp appears to be uncommon but has been reported in areas where canine infection is high. Feline leishmaniosis is presumed to be sandfly-transmitted; however, the vector is unknown and the epidemiology of the disease has not been investigated.

Systemic clinical disease and asymptomatic infection due to $L$. infantum and other species are reported and wild cats have been incriminated as reservoirs for Leishmania spp. in endemic Mediterranean countries. Cutaneous lesions alone have been reported in association with unspecified Leishmania spp in Europe, South America and in the southern USA. The clinical presentation is similar to that seen in dogs although the small number of cases makes the association of infection and clinical signs difficult to interpret. Cutaneous lesions include diffuse areas of alopecia and granulomatous dermatitis of the head, scaling and pinnal dermatitis, ulceration and nodules. Systemic involvement associated with jaundice, vomiting, hepatomegaly, splenomegaly, lymphadenomegaly, membranous glomerulonephritis and granulomatous gastroenteritis has been reported. The same microscopic and molecular tests used for diagnosis of canine leishmaniosis can be used for cats. There are no serological tests validated for cats and therapeutic agents for leishmaniosis in cats have not been investigated. Synthetic pyrethroids should not be used in cats and prevention is limited to decreased exposure to sandfly bites.

\section{Babesiosis}

Babesiosis is caused by protozoan parasites of the genus Babesia that infect erythrocytes and are transmitted by lxodid ticks. The classification of canine babesiae is currently undergoing change as the new species and subspecies are characterised by molecular technology. Two broad groups (large and small babesiae) infect dogs. The Babesia canis group is characterised by larger organisms, and comprises three distinct subspecies. B. canis canis is a moderately pathogenic organism transmitted by the tick Dermacentor reticulatus and it is the major cause of clinical babesiosis in Europe. B. canis vogeli, transmitted by the tick Rhipicephalus sanguineus, is the least pathogenic canine babesia and is widely distributed in the tropical and subtropical regions of most continents including Europe, Asia, Australia, northern Africa and USA. The third subspecies, B. canis rossi, is geographically restricted to southern Africa.

The small babesiae infecting dogs are diverse, of moderate to high pathogenicity, and new species are being increasingly recognised in Europe. Until recently, B. gibsoni was the only recognised babesial organism in this group. It has multiple tick vectors and is distributed

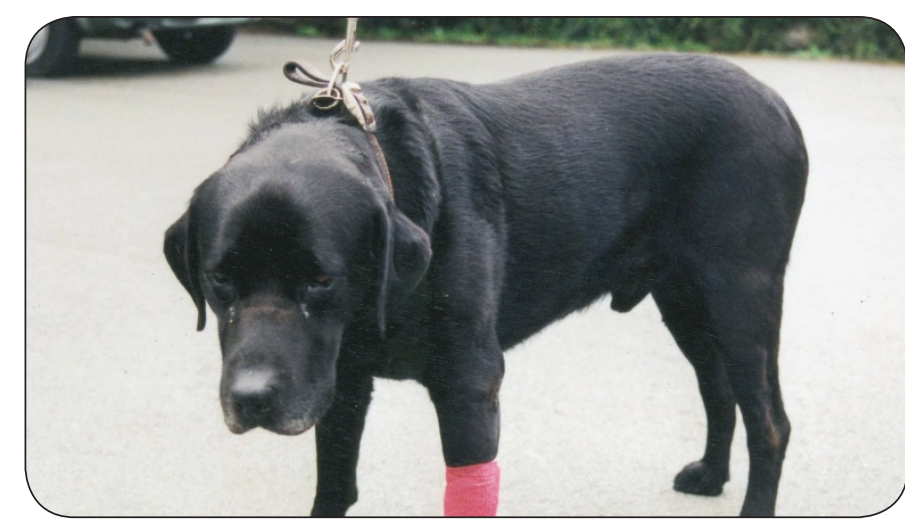

FIGURE I: Five-year-old Labrador retriever recently returned from France with babesiosis, showing severe depression and weakness.

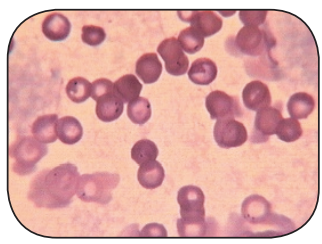

FIGURE 2: Blood smear made from a sample obtained from an ear-tip prick showing B. gibsoni within Diff Quik-stained erythrocytes. Original magnification $\times \mathrm{I}, 000$.

throughout Asia, northern Africa, southern Europe and areas of the USA and Australia. However, other small "babesial" organisms have been identified in dogs with clinical babesiosis, including those in Spain and Hungary that resemble B. microti. In the UK, the tick vectors (D. reticulatus and $R$. sanguineus) are present although localised in distribution; to date, dogs reported with babesiosis have had a history of exposure in other countries of Europe. Non-tick transmission through infected blood transfusions and transplacental transmission have been reported, although uncommonly. Transmission of B. gibsoni from dog to dog through biting has also been suggested. The babesiae infecting companion animals are not considered zoonotic, although the zoonotic potential of the recently identified small babesiae related to $B$. microti remains undetermined. Disease outbreaks of $B$. canis canis in Europe are seasonal, and related to the biology of Dermacentor ticks. Coinfections with Ehrlichia species and Leishmania require consideration.

Pathogenesis and clinical signs

The clinical outcome following infection is dependent on the pathogenicity of the Babesia species and strain, and the age, immune status and breed of the infected dog. Susceptibility amongst greyhounds, Pitbull terriers and Staffordshire Bull Terriers has been reported for B. gibsoni infection, although this may reflect increased chance of infection through biting.

In dogs (Figures I, 2 and 3), the most common clinical presentation in Europe is uncomplicated disease involving an acute onset haemolytic anaemia caused by B. canis canis or B. gibsoni. However, susceptible non-immune travelling dogs may develop hyperacute disease. It is likely that a percentage of dogs develop subclinical infection that may recrudesce at any stage after exposure.

Haemolytic anaemia is the major clinical finding and may involve both intravascular and extravascular haemolysis due to parasite damage to erythrocytes, metabolic changes in erythrocyte membranes leading to increased osmotic fragility, and antibodymediated erythrophagocytosis. Associated clinical signs include pyrexia, haemoglobinuria, mucous membrane pallor, splenomegaly, 

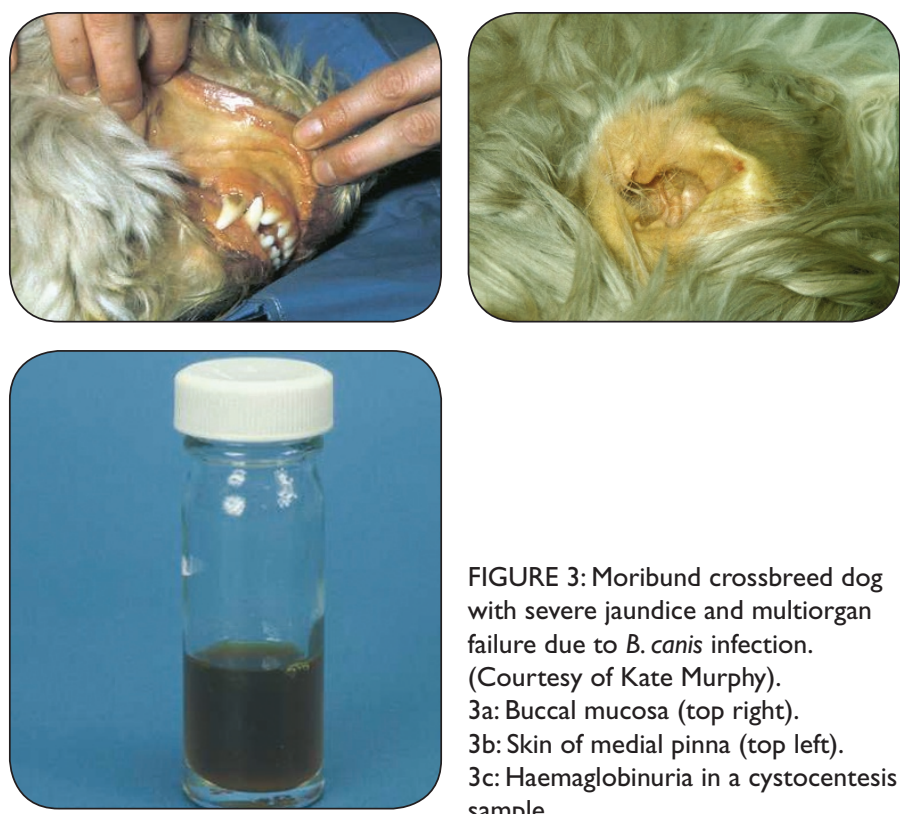

FIGURE 3: Moribund crossbreed dog with severe jaundice and multiorgan failure due to $B$. canis infection. (Courtesy of Kate Murphy). 3a: Buccal mucosa (top right) 3b: Skin of medial pinna (top left). 3 c: Haemaglobinuria in a cystocentesis sample.

lymphadenomegaly, anorexia, vomiting, and lethargy.Thrombocytopenia is common although rarely of sufficient severity to cause bleeding. Blood samples may autoagglutinate in saline and up to $85 \%$ of dogs may be Coombs'-positive. Jaundice can occur but only late in disease and in association with liver dysfunction. Very susceptible dogs infected with B. canis canis may develop multiple organ inflammatory syndrome, hypotensive shock and disseminated intravascular coagulation. Clinical signs may relate to hepatic, renal, and neurological dysfunction. The clinical presentation of babesiosis may be complicated by coinfection with other tick-borne infectious agents. Thrombocytopenia as a result of monocytic ehrlichiosis may complicate babesia-induced haemolytic anaemia with haemorrhagic diatheses.

\section{Diagnosis}

In dogs with appropriate clinical signs and a history of exposure, blood smears stained by Romanowsky or Wright's methods should be examined for intraerythrocytic organisms. Smears should be prepared from blood obtained from pricking an ear tip as this increases the chance of observing parasitised erythrocytes. However, sensitivity is reduced by low level and fluctuating parasitaemia as well as by the difficulty in identifying the small babesia species. Serological tests (IFA or ELISA) for some babesia species have been developed but they are not easily commercially available in Europe and have problems with poor sensitivity and specificity. PCR testing of blood samples for all Babesia species is highly sensitive and specific for infection and real time PCR will be available for quantification of microbial load for monitoring purposes.

\section{Treatment and contro}

The treatment of choice for $B$. canis canis and $B$. canis vogeli is imidocarb dipropionate although there is not a licensed product available in Ireland or UK at present (Table I). This treatment is ineffective against $B$. gibsoni, which is more successfully treated with a combination of ataquarone and azithromycin (Table I). Other drugs used, but left in reserve, include phenamidine isethionate, pentamidine isethionate and diminazene aceturate. Infected animals may become chronic carriers despite therapy, although the degree to which this occurs when exposure is limited in non-endemic countries is not confirmed. Supportive therapy for haemolytic anaemia (including fluids and cross-matched blood transfusions) may be required and, although controversial, a short course of glucocorticoids may be appropriate to counter the immune-mediated component of the disease.

Prevention of babesiosis is dependent on control of the vector ticks using fipronil, permethrin or deltamethrin. Owners should be encouraged to examine animals daily in order to remove ticks before attachment. There is on-going European research into development of B. canis vaccines.

Feline babesiosis

Feline babesiae are poorly characterised. B. felis is primarily found in southern Africa and a separate subspecies of $B$. canis has recently been reported in Israeli cats. A report of a large babesia species occurring in a cat has been published in India. Separate babesiae infect wild Felidae. The tick vectors are unknown. In South Africa, B. felis infection has no breed or gender predisposition. The clinical presentation is that of chronic regenerative anaemia but adaptation of the cat to the anaemic state means that clinical signs may only manifest with stress. The most commonly recommended drug for treatment of feline babesiosis in South Africa is primaquine phosphate (Table I).

\section{Canine monocytic ehrlichiosis}

Monocytic ehrlichiosis due to Ehrlichia canis has a global distribution in tropical and semi-tropical regions throughout the world and its distribution follows that of its vector, the tick Rhipicephalus sanguineus. This tick is present in focal areas of the UK, including quarantine kennels and has adapted well to households in which there are dogs. Monocytic ehrlichiosis is now endemic in central Europe including Austria, Switzerland and southern Germany, as well as the southern European countries.

\section{Pathogenesis and clinical signs}

Following infection, there is an acute phase of illness (eight to 20 days) followed by clinical remission, which may last for years. In susceptible animals, a waxing and waning syndrome of chronic ehrlichiosis develops associated with bone marrow dysplasia and other immunemediated organ damage. Coinfection with Babesia spp. and L. infantum is common in endemic areas. In acute canine monocytic ehrlichiosis, clinical signs include fever, anorexia, and lymphadenopathy combined with a bleeding disorder. Petechiation (Figure 4), epistaxis, intraocular bleeding are common due to immune-mediated thrombocytopenia and platelet dysfunction. Dogs, which are subclinically infected, may relapse intermittently with immunosuppressive episodes. In chronic disease, hypergammaglobulinaemia is a feature as are signs of bone marrow damage. This may be restricted to platelet precursors but varying degrees of non-regenerative anaemia, thrombocytopenia and leucopenia may develop in susceptible animals. In the German shepherd breed, pancytopenia may be irreversible and fatal.

\section{Diagnosis}

Diagnosis of monocytic ehrlichiosis is based on appropriate clinical findings and microscopic identification of aggregations of ehrlichial organisms (morulae) in monocytes on a Wright's stained capillary blood smear (from a pricked eartip), buffy coat smear or splenic 
aspirate. Many serological tests are now available but there is considerable cross-reaction with other ehrlicial and rickettsial species and a rising titre is required to confirm active infection. Persistent antibody production makes monitoring of therapy and interpretation difficult. Species-specific PCR tests on EDTA blood and splenic aspirates are sensitive and specific, they distinguish between infection and exposure, and they identify cases early in disease progression.

Treatment and control

Supportive therapy with fluids and blood transfusions may be required. If life-threatening thrombocytopenia is present, glucocorticoid therapy has some rationale as the pathogenesis of the thrombocytopenia has an immune-mediated component. Doxycycline or oxytetracycline therapy is the treatment of choice for ehrlichiosis (Table I) for 21 to 28 days. Imidocarb dipropionate can be given for resistant infections although its efficacy is debatable. At present, vaccination is not available. Prophylaxis for travelling dogs involves tick control using fipronil, permethrin or deltamethrin and owners should be encouraged to examine dogs daily to remove ticks prior to attachment.

Feline monocytic ehrlichiosis

Feline ehrlichiosis associated with inclusions in mononuclear cells has been reported in cats with seropositivity to and PCR-positivity for $E$. canis. As in other species, monocytic ehrlichiosis in cats is presumed to be tick-transmitted. Clinical signs with naturally occurring disease include intermittent fever, anorexia, weight loss, vomiting, diarrhoea, upper respiratory signs and muscle pain. Clinicopathological findings include anaemia, thrombocytopenia, leucopenia and hyperglobulinaemia. Statistically significant associations between clinical signs and positive serology are more limited. Definite diagnosis of feline ehrlichiosis is made by demonstration of intramonocytic inclusions in, or PCR of, a peripheral blood sample.

Although controlled therapeutic trials are lacking, administration of doxycycline or tetracycline (Table I) is the treatment of choice for feline ehrlichiosis and clinical response is reported to be excellent. Control of vectors offers the most appropriate method of prophylaxis.

\section{Canine anaplasmosis (canine cyclic thrombocytopenia)} Canine cyclic thrombocytopenia, caused by Anaplasma (Ehrlichia) platys, occurs in several southern European countries, Israel, Taiwan, Venezuela and, most recently, Australia. This species of Anaplasma has tropism for platelets and it is assumed that it is transmitted by $R$. sanguineus. Pathogenicity is generally low but $A$. (E.) platys infection may play a role in coinfection with other arthropod borne diseases such as Babesia or Ehrlichia species which are also transmitted by Rhipicephalus. Mild to moderate thrombocytopenia occurs at intervals of two to three weeks and is primarily asymptomatic unless the dog undergoes surgery or has a concurrent bleeding disorder. However, there are reports of a more pathogenic strain from Greece and the Middle East. Platelet inclusions are a useful means of diagnosis, although species-specific PCR tests have now been developed. Considering the cyclic nature and low levels of the parasitaemia, molecular diagnosis offers a more sensitive alternative to cytology. IFA tests have been developed but are not commercially

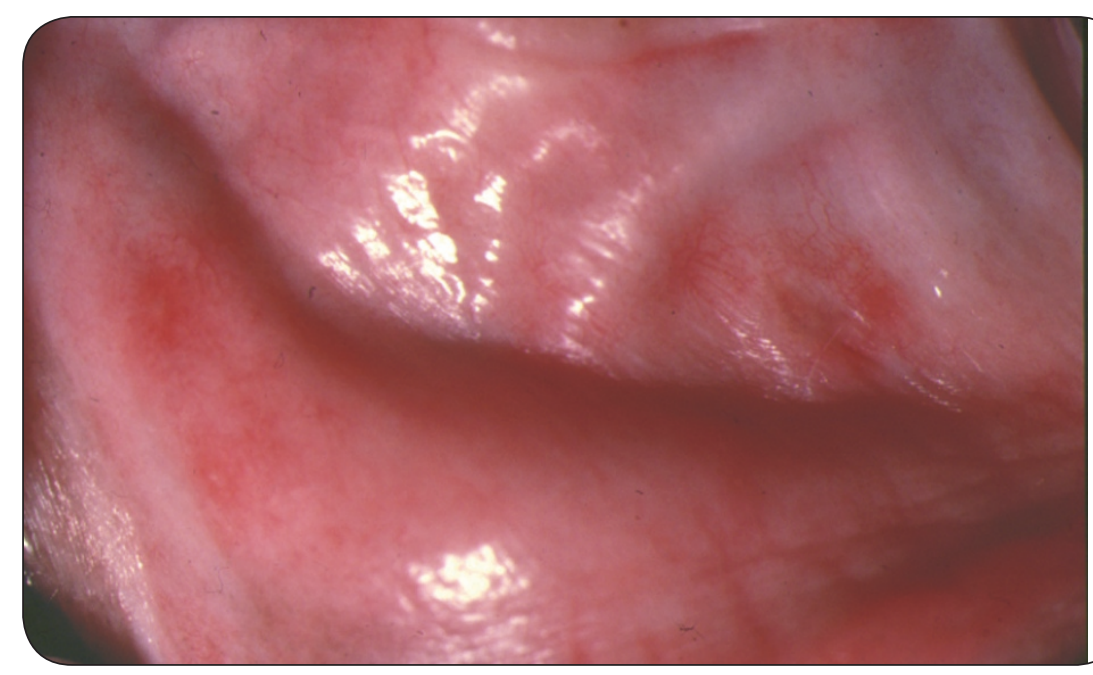

FIGURE 4: Buccal mucosal petechiation in a clinical case of ehrlichosis in a dog (E. canis).

available. Given the asymptomatic nature of the disease, treatment may not be necessary although infection is responsive to tetracyclines. Vector control, using fipronil, permethrin or deltamethrin, is the most appropriate means of prevention.

\section{Heartworm disease (dirofilariosis)}

Heartworm infection and disease is caused by the filarial nematode, Dirofilaria immitus and is transmitted by mosquitoes of many species. Dogs are the primary hosts for $D$. immitus.

Heartworm infection in cats, although rarely associated with latency, is being increasingly recognised in areas where the infection rate in dogs is high. Non-patent human infections may occur rarely. Canine dirofilariosis is endemic in southern Europe, the Americas, Australia, Africa and Asia. In Europe, it is prevalent in focal areas of Portugal, Spain, southern France, Italy, Greece and other countries of the Mediterranean. At present, heartworm infection is not endemic in northern Europe despite the fact that competent vector species are present, because larval maturation in the mosquito is inhibited where mean summer temperatures are low. At present, areas of temperate continental Europe that have higher summer temperatures are more at risk for new outbreaks than the UK or Ireland. Another risk for establishment is the potential for genetic adaptation for maturation at cooler temperatures. Mosquitoes ingest the LI larvae (microfilariae) with a blood meal from an infected dog and, after an obligatory period of development, the infective L3 larval stage is injected back into a susceptible host. Larvae undergo several moults and migrate through host tissues to the right pulmonary artery where they mature to adults six to eight months after infection. Adult female heartworms are viviparous and produce LI larvae directly into the systemic circulation. Adult heartworms can live for five to six years, and the microfilariae for up to two years.

\section{Pathogenesis and clinical signs}

Adult worms in the pulmonary artery cause thickening of the vessel endothelium and smooth muscle layer. Thromboembolism is initiated, causing obstruction of smaller pulmonary vessels and periarterial inflammation. Development of pulmonary hypertension may result in exercise intolerance, syncope and weight loss. In sensitised animals, 


\section{TABLE I: Recommended treatments for exotic arthropod-borne diseases of dogs and cats}

\begin{tabular}{|c|c|c|c|c|}
\hline Disease & $\begin{array}{l}\text { Generic } \\
\text { product }\end{array}$ & $\begin{array}{l}\text { Recommended } \\
\text { dose rates }\end{array}$ & $\begin{array}{l}\text { Interval and duration } \\
\text { of therapy }\end{array}$ & Notes/toxicity \\
\hline LEISHMANIOSIS & $\begin{array}{l}\text { Allopurinol (purine analogue) } \\
\text { Meglumine antimonate } \\
\text { (pentavalent antimonial) }\end{array}$ & $\begin{array}{l}\text { Dogs: } 10-15 \mathrm{mg} / \mathrm{kg} \text { PO } \\
\text { Dogs: } 100 \mathrm{mg} / \mathrm{kg} \mathrm{SC}\end{array}$ & $\begin{array}{l}\text { q } 12-24 \mathrm{~h} \\
\text { q24-48h for } 20-40 \text { days }\end{array}$ & $\begin{array}{l}\text { Increase uric acid } \\
\text { levels } \\
\text { Pain, swelling at } \\
\text { injection site, } \\
\text { renal dysfunction, } \\
\text { increased liver } \\
\text { enzymes. }\end{array}$ \\
\hline \multicolumn{5}{|l|}{ BABESIOSIS } \\
\hline B. canis canis & Imidocarb dipropionate & $5.0-6.6 \mathrm{mg} / \mathrm{kg}$ IM or SC & Once, then repeat $2-3$ weeks & Uncommon salivation, \\
\hline B. gibsoni & $\begin{array}{l}\text { Ataquavone in combination } \\
\text { with azithromycin }\end{array}$ & $\begin{array}{l}13.3 \mathrm{mg} / \mathrm{kg} \mathrm{PO}+ \\
10 \mathrm{mg} / \mathrm{kg} \mathrm{PO}\end{array}$ & $\begin{array}{l}\text { q8hrs }+ \\
\text { q24hrs for } 10 \text { days }\end{array}$ & $\begin{array}{l}\text { lacrimation, diarrhoea, } \\
\text { vomiting, }\end{array}$ \\
\hline B. felis & Primiquine phosphate & $0.3 \mathrm{mg} / \mathrm{kg} \mathrm{PO}$ & q24hrs for 14 days & $\begin{array}{l}\text { muscle tremor, } \\
\text { pain on injection. }\end{array}$ \\
\hline \multicolumn{5}{|l|}{ EHRLICHIOSIS } \\
\hline ANAPLASMOSIS & Doxycycline & $\begin{array}{l}\text { Dogs: } 5-10 \mathrm{mg} / \mathrm{kg} \text { PO } \\
\text { Cats: } 5 \mathrm{mg} / \mathrm{kg} \text { PO }\end{array}$ & q |2-24h for $|4-2|$ days & $\begin{array}{l}\text { Stain teeth in first } \\
\text { month of life and } \\
\text { in last trimester }\end{array}$ \\
\hline & Oxytetracycline & $\begin{array}{l}\text { Dogs and cats } 7-11 \mathrm{mg} / \mathrm{kg} \\
\text { IM,SC } \\
\text { Dogs } 25 \mathrm{mg} / \mathrm{kg} \text { PO }\end{array}$ & $\begin{array}{l}\text { q8h for }|4-2| \text { days } \\
\text { q8h for }|4-2| \text { days }\end{array}$ & $\begin{array}{l}\text { pregnancy. Anorexia } \\
\text { especially in cats, } \\
\text { vomiting, diarrhoea. }\end{array}$ \\
\hline \multicolumn{5}{|l|}{ DIROFILARIOSIS } \\
\hline & Melarsomine & $\begin{array}{l}\text { Protocol A } 2.2 \mathrm{mg} / \mathrm{kg} \text { IM } \\
\text { or } \\
\text { Protoocol B } 2.5 \mathrm{mg} / \mathrm{kg} \text { IM }\end{array}$ & $\begin{array}{l}\text { Once then again at } 3 \mathrm{~h} \\
\text { Once then repeat at } 24 \mathrm{~h}\end{array}$ & $\begin{array}{l}\text { Dosing protocol } \\
\text { dependant on } \\
\text { clinical classification. } \\
\text { Subclinical disease: } \\
\text { Protocols A or B } \\
\text { Moderate disease: } \\
\text { Protocol B } \\
\text { Severe: Single } 2.5 \mathrm{mg} / \mathrm{kg} \\
\text { treatment followed by } \\
\text { Protocol B I-2 } \\
\text { months later. }\end{array}$ \\
\hline
\end{tabular}

eosinophilic pneumonitis can be induced by parasite antigen without major arterial changes, and immunological destruction of circulating microfilariae can occur. Clinical signs may only develop when animals undergo cardiovascular stress, have heavy parasite burdens or sensitivity to parasite antigens. Early signs are exercise intolerance and coughing. Dyspnoea, weight loss and pulmonary haemorrhage may occur in more severe cases. Signs of right-sided cardiac failure (jugular distension, hepatomegaly) may occur later in disease or severe infestations. A rare (caval) syndrome may occur when susceptible animals are infected with high worm burdens all maturing at a similar time, causing obstruction of the vena cava and the pulmonary outflow tract. Clinical signs are characterised by intravascular haemolysis due to erythrocyte fragmentation, collapse, hypotension, pale mucous membranes, tricuspid murmur, dysrhythmias, haemoglobinuria, hepatomegaly and sudden death.

\section{Diagnosis}

Diagnosis of dirofilariosis involves microscopic examination of EDTA blood for microfilariae using the modified Knott's filter test. Other species of non-pathogenic filarial worms (Dipetalonema reconditum and Dirofilaria repens) produce microfilaraemia and may give false-positive results unless proper parasitological identification is available. $D$. immitiis infections caused by single sex infections, immature burdens, or immune-mediated destruction of microfilaria result in falsenegative results. ELISA testing based on detection of $D$. immitis uterine antigen is available from most clinical diagnostic laboratories, and is specific and sensitive except in single sex infections. Antibody testing for $D$. immitis lacks specificity for canine patients. Diagnostic imaging of clinical cases reveals tortuous, pruned arteries (especially of the right caudal lobe), interstitial and alveolar infiltrates and right-sided cardiac enlargement with or without a pulmonary artery bulge. Echocardiography may allow visualisation of the adult worms and is the diagnostic method of choice in caval syndrome.

Treatment and control

Symptomatic treatment of heart failure and judicious management of allergic pulmonary disease with prednisolone is appropriate prior to administration of parasiticidal drugs. Prednisolone should be 
withdrawn 48 hours prior to parasiticidal therapy to increase efficacy of adult nematode kill. Parasiticidal therapy is associated with potential adverse effects, particularly pneumonitis and thromboembolism, following death of the adult worms. At present, melarsomine is the adulticidal drug of choice in Europe (Table I). Dogs should be cagerested after therapy to minimise thromboembolism, and prednisolone can be reintroduced to control inflammatory complications. Prognosis in early to moderately severe heartworm disease is good. Use of a microfilaricidal drug following adulticide therapy is debatable in non-endemic areas as there is minimal risk of transmitting infection. However, persistent microfilaraemia may incite immune-mediated damage and ivermectin or milbemycin can be used as microfilaricides. Caval syndrome is an emergency and adult worms must be removed with forceps from the vena cava and heart via jugular venotomy. Survival rates are less than $50 \%$.

Prevention is primarily achieved by administration of drugs that kill migrating larvae and drugs available in Europe include selemectin and milbemycin oxime alone and in combination with other drugs (Table I). Dogs travelling to endemic countries should begin preventative therapy seven to ten days prior to travel and the treatment duration should ensure that they are covered completely while they are abroad. Where there is any possibility that dogs may already be infected, testing should be performed as adverse or unexpected reactions may occur if preventative medications are administered to infected cases. Routine prophylaxis for puppies can start from six to eight weeks of age.

\section{Feline dirofilariosis}

Cats may develop non-patent infections in areas of high endemicity They have low worm burdens without production of microfilariae. Immune-mediated pulmonary disease may result and clinical signs include dyspnoea, coughing, weight loss, vomiting and sudden death. Non-patent infection limits the usefulness of diagnostic tests and there is a role for antibody testing in feline patients with single or low worm burdens. Echocardiography is particularly useful as part of the diagnostic regime in feline dirofilariosis. Parasiticidal treatment is problematic as the arsenical preparations used in dogs are particularly toxic for cats. Symptomatic treatment of associated pulmonary disease with corticosteroids and cage rest is recommended. Selemectin and milbemycin are licensed for cats for heartworm prevention in the UK.

\section{Further reading}

Baneth, G. and Shaw, S.E. (2002). Chemotherapy of canine leishmaniosis. Veterinary Parasitology 106: 31 5-324.

Bowen-Davies, J. and Lowings, P. (2000). Current perspectives on rabies I.The biology of rabies and rabies-related viruses. In Practice 22: $118-124$.

Bowen-Davies, J. and Lowings, P. (2000). Current perspectives on rabies 2. Review of classical rabies and its control. In Practice 22: I70-175.

Ciaramella, P., Oliva, G., de Luna, R., Gradoni, L., Ambrosio, R., Cortese, L., Scalone, A. and Persechino, A. (1997). A retrospective clinical study of canine leishmaniasis in 150 dogs naturally infected by Leishmania infantum. Veterinary Record I4I: 539-543.

Executive Board of the American Heartworm Society (2004).
Guidelines for the diagnosis, prevention and management of heartworm (Dirofilaria immitus) infection in dogs and cats. http:// www.heartwormsociety.org

Ferasin, L. (2004). Heartworm disease - potential health risks for the travelling pet. In Practice 26: 350-357.

Harrus, S., Aroch, I., Lavy, E. and Bark, H. (1997). Clinical manifestations of infectious canine cyclic thrombocytopenia. Veterinary Record I 4I: 247-250.

Harrus, S., Kass, P.H., Klement, E. and Waner, T. (1997). Canine monocytic ehrlichiosis: A retrospective study of 100 cases, and an epidemiological investigation of prognostic factors for the disease. Veterinary Record I4 I: 360 -363.

Homer, M.J., Aguilar-Delfin, I., Telford, S.R., Krause, P.J. and Persing, D.H. (2000). Babesiosis. Clinical Microbiological Reviews I3: 451-469.

Koutinas, A. F., Polizopoulou, Z. S., Saridomichelakis, M. N., Argyriadis, D., Fytianou, A. and Plevraki, K.G. (1999). Clinical considerations on canine visceral leishmaniasis in Greece: a retrospective study of 158 cases (1989-1996). Journal of the American Animal Hospital Association 35: 376-383.

Miller, M. (1998). Feline dirofilariasis. Clinical Techniques in Small Animal Practice 13:99-109.

Shaw, S.E., Birtles, R.J. and Day, M.J. (200I). Arthropod-borne infectious diseases of cats. Journal of Feline Medicine and Surgery 3: 193-209.

Shaw, S.E., Day, M.J., Birtles, R.J. and Breitschwerdt, E. (200I). Tick-transmitted infectious diseases of dogs. Trends in Parasitology I7: 74-80.

Shaw, S.E., Lerga, A.I., Williams, S., Beugnet, F., Birtles, R.J., Day, M.J. and Kenny, M.J. (2003). Review of exotic infectious diseases in small animals entering the United Kingdom from abroad diagnosed by PCR. Veterinary Record I 52: I76- 177.

Stubbs, C.J., Holland, C.J., Reif, J.S., Bruns, C. and Lappin, M.R. (2000). Feline ehrlichiosis. Compendium of Continuing Education for Practising Veterinarians 22: 307-317.

Trees, A. and Shaw, S.E. (1999). Imported diseases in small animals. In Practice 10: 482-491. 